EPJdirect (2018), to be processed by the editorial

EPJdirect

electronic only

(C) Springer-Verlag 2018

\title{
Production and Decay of Excited Electrons at the LHC
}

O.Çakır ${ }^{1}$, C. Leroy ${ }^{2}$, R. Mehdiyev ${ }^{2,3}$, A. Belyaev ${ }^{4,5}$

1 Ankara University, Faculty of Sciences, Department of Physics, 06100, Tandogan, Ankara, Turkey.

2 Université de Montréal, Département de Physique, Montréal, H3C 3J7, Canada.

${ }^{3}$ on leave of absence from Institute of Physics, Azerbaijan National Academy of Sciences, 370143, Baku, Azerbaijan.

4 Department of Physics, Florida State University, Tallahassee, FL, USA.

${ }^{5}$ on leave of absence from Skobeltsyn Institute for Nuclear Physics, Moscow State University, 119 899, Moscow, Russian Federation

\begin{abstract}
We study single production of excited electrons at the CERN LHC through contact interactions of fermions. Subsequent decays of excited electrons to ordinary electrons and light fermions via gauge and contact interactions are examined. The mass range accessible with the ATLAS detector is obtained.
\end{abstract}

PACS: $12.60 . \mathrm{Rc}, 13.85 . \mathrm{Rm}$

\section{Introduction}

One of the natural explanations for the existence of the fermionic generations is the compositeness [1] of the known leptons and quarks, sharing common constituents (preons). According to this approach, a quark or lepton might be a bound state of three fermions [2] or a fermion and a boson [3. In many models along this line, quarks and leptons are composed of a scalar and a spin- $1 / 2$ preon. Composite models [1] predict a rich spectrum of excited states 1, 4] of known particles. Naturally, in these models spin- $1 / 2$ is assigned to the lowest lying radial and orbital excitations. Searching for the spin of the lowest lying fermionic excitations could thus give a rather direct clue to the underlying preon structure. Therefore, it is important to conduct experiments which will probe possible substructure of leptons and quarks and test the variety of preonic models.

Exchange of preons may lead to contact interactions between quarks and leptons. In this sense, it is conceivable that the standard model (SM) is just the low energy limit of a more fundamental theory which is characterized by a large mass scale $\Lambda$. The existence of four-fermion contact interactions would be a signal of new physics beyond the SM. If the experimental energy scale is high enough, the nature of this new physics can be probed. It is expected that the next generation of hadron colliders like the LHC which will achieve very high centre of mass energies will extend the search for composite states. In particular, contact interactions may be an important source for excited lepton production at the CERN LHC. Updated lower limits on the scale of compositeness $\Lambda$ are 
given by the ALEPH Collaboration, $\Lambda>6.2 \mathrm{TeV}[5]$ and D0 Collaboration $\Lambda>4.2 \mathrm{TeV}[6]$. Lower mass limits for excited lepton mass are given by the ZEUS Collaboration, $m_{e^{\star}}>200 \mathrm{GeV}\left[7\right.$ and the OPAL Collaboration, $m_{e^{\star}}>306$ $\mathrm{GeV}$ 8.

In the present study, single production of excited electrons is simulated through contact interactions at LHC energies.

The excited muon production is similar to $e^{\star}$ production at hadron colliders. However, with a good $e / \mu$ separation, $\mu^{\star}$ can be easily detected. But overall statistical significances for $\mu^{\star}$ should be very similar to an excited electron production case.

This work is a continuation of previous work devoted to the study of the excited quark production with subsequent decay to a quark and photon [9], a quark and gluon [10] and a quark and $W / Z[11$.

\section{Effective Lagrangian}

In phenomenological models, it is assumed that any theory of compositeness at large mass scale must have a low energy limit that preserves the symmetries of the SM. If quarks and leptons are composite at the energy scale $\Lambda$, the strong forces binding their constituents induce flavor-diagonal contact interactions, which have significant effects at subprocess energies well below $\Lambda$. Contact interactions between quarks and leptons may appear as the low energy

limit of the exchange of heavy particles. At sufficiently high energies excited fermions could be produced directly. They should form weak iso-doublets and carry electromagnetic charges similar to those of the ordinary fermions. We will assume that the excited leptons have spin and isospin $1 / 2$ to limit the number of parameters.

\subsection{Production of excited electrons}

Excited electrons may couple to ordinary quarks via contact interactions resulting from preon interactions. For energies below the compositeness scale $\Lambda$, these interactions can be described by an effective four-fermion Lagrangian [4]

$$
L_{C}=\frac{g_{\star}^{2}}{2 \Lambda^{2}} j^{\mu} j_{\mu}
$$

with

$$
j_{\mu}=\eta_{L} \bar{f}_{L} \gamma_{\mu} f_{L}+\eta_{L}^{\prime} \bar{f}_{L}^{\star} \gamma_{\mu} f_{L}^{\star}+\eta_{L}^{\prime \prime} \bar{f}_{L}^{\star} \gamma_{\mu} f_{L}+(L \rightarrow R)+\text { h.c. }
$$

where the coupling $g_{\star}^{2}=4 \pi ; \eta_{L}$ and $\eta_{R}$ are coefficients for left-handed and right-handed currents, respectively. Here we assume $\eta_{L}=\eta_{L}^{\prime}=\eta_{L}^{\prime \prime}=1$ and $\eta_{R}=\eta_{R}^{\prime}=\eta_{R}^{\prime \prime}=0$, for simplicity.

At the CERN LHC pp collider, excited electrons can be produced either singly $q \bar{q} \rightarrow l \bar{l}^{\star}, l^{\star} \bar{l}$ or in pairs $q \bar{q} \rightarrow l^{\star} \bar{l}^{\star}$ through contact interactions as shown in Fig. 1] 

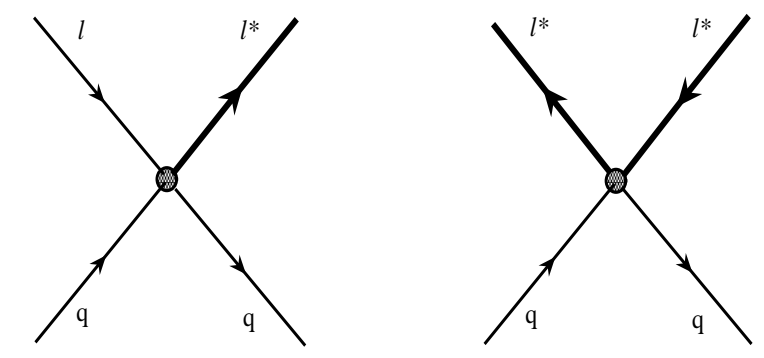

Fig. 1. Contact interactions of excited electrons $\left(l^{\star}\right)$ with quark $(q)$ and leptons (l)

The partonic cross sections for excited electron production through contact interactions are given by 4

$$
\begin{gathered}
\widehat{\sigma}\left(q \bar{q} \rightarrow l \bar{l}^{\star}, l^{\star} \bar{l}\right)=\frac{\pi}{6 \widehat{s}}\left(\frac{\widehat{s}}{\Lambda^{2}}\right)^{2}\left(1+\frac{v}{3}\right)\left(1-\frac{m_{\star}^{2}}{\widehat{s}}\right)^{2}\left(1+\frac{m_{\star}^{2}}{\widehat{s}}\right) \\
\widehat{\sigma}\left(q \bar{q} \rightarrow l^{\star} \bar{l}^{\star}\right)=\frac{\pi \widetilde{v}}{12 \widehat{s}}\left(\frac{\widehat{s}}{\Lambda^{2}}\right)^{2}\left(1+\frac{\widetilde{v}^{2}}{3}\right)
\end{gathered}
$$

where

$$
v=\frac{\widehat{s}-m_{\star}^{2}}{\widehat{s}+m_{\star}^{2}}, \quad \widetilde{v}=\left(1-4 \frac{m_{\star}^{2}}{\widehat{s}}\right)^{1 / 2}
$$

where $\widehat{s}$ denotes the Mandelstam variable for the subprocess, the centre of mass energy.

Cross sections for single and pair production of excited electrons through contact interactions are shown in Fig. 2 Since $l^{\star} \overline{l^{\star}}$ pair production requires larger centre of mass energy than single $l \bar{l}^{\star}$ production, it is less favored.

Cross section values presented in this paper were calculated using CTEQ5L parton distribution function (PDF) 12. Other PDF's were tried: GRV94 LO 13 and CTEQ3L 14 differ in their cross-section prediction by about $20 \%$ and $30 \%$, respectively. These PDF's extend slightly the mass reach for excited electrons at the LHC.

One should notice that excited leptons can also be produced via gauge interactions (see the effective Lagrangian in the next subsection). Gauge interactions can give rise to $\ell^{*} \ell^{*}, \ell \ell^{*}, \ell^{*} \nu$ signatures. The study of such scenario has been done in [15. However, since those processes involve electromagnetic or electroweak couplings they contribute to less than $1 \%$ compared to the excited lepton production rate via contact interactions.

In the same time contact interactions will modify the Drell-Yan(DY) dilepton production process and one should definitively check how big are deviations from the SM [15]. It is important to notice that the sensitivity of the LHC to the compositeness scale in the Drell-Yan channel is very high - for an integrated luminosity of $100 \mathrm{fb}^{-1}$, one has $\Lambda<30 \mathrm{TeV}$ at 5 sigma level limit ! [22] 


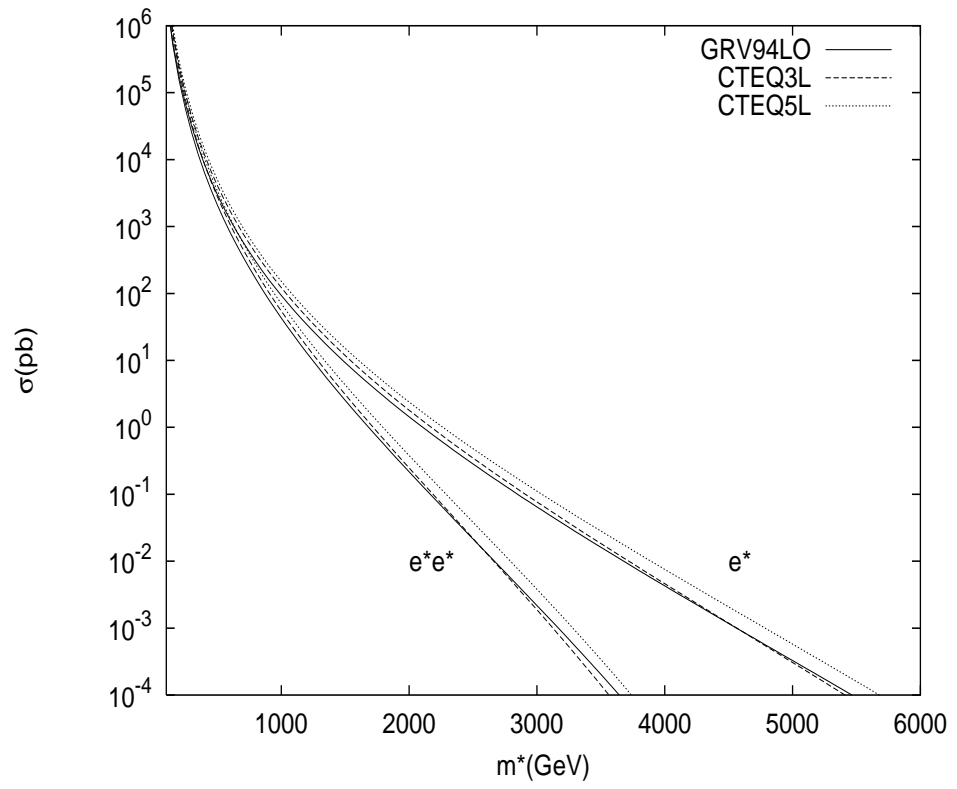

Fig. 2. Cross sections for single and pair production of excited electrons through contact interactions at LHC $\left(\Lambda=m_{\star}\right)$ calculated using different PDFs

This means that, for the range of $\Lambda \sim 30 \mathrm{TeV}$ we consider here, the signal in the DY channel from contact interactions will be unavoidably large! Therefore our study should be considered as the study not for the determination of the limits on $\Lambda$ (which can be done much better using DY channel) but as the study of the excited electron production at the LHC. One should stress the big complementary role of the DY channel, however, if one would observe signal from the excited electron production, the signal from contact interactions should also be present in DY channel too.

One should notice also that the "charged current" contact term like $u d \rightarrow$ $e \nu$ is not forbidden by $U(1) \times S U(2) L \times S U(3)$ symmetry and can give rise to $l^{*} \nu$ production in contact interactions at the LHC. We do not consider this effective interaction in our study.

\subsection{Decay via gauge interactions}

The effective Lagrangian which describes the transition between excited and ground states via gauge interactions is given by [4]

$$
L_{G}=\frac{1}{2 \Lambda} \bar{l}_{R}^{\star} \sigma^{\mu \nu}\left(g f \frac{\tau}{2} W_{\mu \nu}+g^{\prime} f^{\prime} \frac{Y}{2} B_{\mu \nu}\right) l_{L}+\text { h.c. }
$$

where $W_{\mu \nu}$ and $B_{\mu \nu}$ are the field strength tensors of the $\mathrm{SU}(2)$ and $\mathrm{U}(1)$ gauge fields with the gauge structure constants $\tau$ and $Y$, respectively; the factors $f$ and $f^{\prime}$ describe the effective deviations from the SM coupling constants; $g$ and 


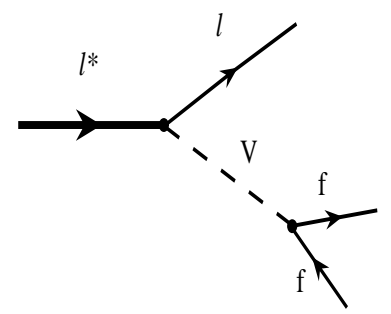

Fig. 3. Decay of excited lepton $\left(l^{\star}\right)$ into a lepton $(l)$ and a pair of fermions $(f)$ via gauge interactions mediated by the vector boson $(\mathrm{V})$.

$g^{\prime}$ are the corresponding gauge coupling constants. An excited electron can then decay via gauge interactions to a gauge boson and an ordinary lepton.

Here we assume that excited electrons have a mass larger than the $W$ and $Z$ boson masses and the main decay mode via gauge interaction will be two-body decays (Fig. B1).

The decay widths:

$$
\begin{aligned}
& \Gamma_{G}\left(e^{*} \rightarrow \gamma e\right)=\frac{\alpha_{\gamma}}{4} \frac{m_{\star}^{3}}{\Lambda^{2}} f_{\gamma}^{2} \\
& \Gamma_{G}\left(e^{*} \rightarrow W \nu\right)=\frac{\alpha_{W}}{4} \frac{m_{\star}^{3}}{\Lambda^{2}} f_{W}^{2}\left(1-\frac{m_{W}^{2}}{m_{\star}^{2}}\right)^{2}\left(1+\frac{m_{W}^{2}}{2 m_{\star}^{2}}\right) \\
& \Gamma_{G}\left(e^{*} \rightarrow Z e\right)=\frac{\alpha_{Z}}{4} \frac{m_{\star}^{3}}{\Lambda^{2}} f_{Z}^{2}\left(1-\frac{m_{Z}^{2}}{m_{\star}^{2}}\right)^{2}\left(1+\frac{m_{Z}^{2}}{2 m_{\star}^{2}}\right)
\end{aligned}
$$

where $f_{\gamma}=f T_{3}+f^{\prime} Y / 2, f_{W}=f / \sqrt{2}$ and $f_{Z}=f T_{3} \cos ^{2} \theta_{W}-f^{\prime}(Y / 2) \sin ^{2} \theta_{W}$, and $T_{3}$ and $Y$ denotes the third component of the weak isospin and hypercharge of $e^{\star}$, respectively. The structure constants are defined as $\alpha_{W}=\alpha / \sin \theta_{w}$ and $\alpha_{Z}=\alpha_{W} / \cos \theta_{w}$, where $\theta_{w}$ is the Weinberg angle. For $m_{\star} \gg m_{W}, m_{Z}$ we neglect $m_{W}^{2} / m_{\star}^{2}$ and $m_{Z}^{2} / m_{\star}^{2}$ terms, and the total width for gauge interaction decay can be obtained as follows

$$
\Gamma_{G}\left(e^{*} \rightarrow a l l\right) \simeq \frac{1}{4} \frac{m_{\star}^{3}}{\Lambda^{2}}\left(\alpha_{\gamma} f_{\gamma}^{2}+\alpha_{W} f_{W}^{2}+\alpha_{Z} f_{Z}^{2}\right)
$$

where the parameters $f_{\gamma}, f_{W}$ and $f_{Z}$ simply reduce to the approximate values $-1(0), 0.707(0.707)$ and $-0.269(-0.5)$ for $f=f^{\prime}=1\left(f=-f^{\prime}=-1\right)$, respectively.

The present analysis will assume $f=f^{\prime}=1$, but the results can easily be reinterpreted for different values of these parameters, accounting for the change in branching ratio and intrinsic width.

\subsection{Decay via contact interactions}

The decay width of a fermion via contact interactions ( three-body decay) is given by

$$
\Gamma_{C}\left(e^{\star} \rightarrow e+f \bar{f}\right)=\frac{1}{96 \pi} N_{C} S \frac{m_{\star}^{5}}{\Lambda^{4}}
$$


where $N_{C}$ is the number of colours of the fermions $\left(N_{C}=3(1)\right.$ for quarks(leptons)) and $S$ is a factor given by

$$
\begin{array}{lll}
S=1 & \text { for } & f=q, l \neq e \\
S=2 & \text { for } & f=e
\end{array}
$$

We present decay widths and branching ratios for gauge and contact interactions in Table 1 for $\Lambda=m_{\star}$ and for the case when we fix $\Lambda$ at $6 \mathrm{TeV}$ scale. The choice of $\Lambda=6 \mathrm{TeV}$ is motivated by the fact that the case of $\Lambda=m_{\star}$ looks unnatural from the theoretical point of view. The effective energy of the parton-level collision $(\sqrt{\hat{s}})$ at the LHC is of the order of several TeV. In case $\sqrt{\hat{s}}>\Lambda$ the validity of series expansion of the effective Lagrangian in terms of $\sqrt{\hat{s}} / \Lambda$ is arguable, at least for $m_{\star}$ values below $1 \mathrm{TeV}$. With respect to this fact, the choice of $\Lambda \simeq O(10 \mathrm{TeV})$ is safer and theoretically motivated. Here we will take $\Lambda=6 \mathrm{TeV}$, corresponding to the ALEPH limit [5], as a reference.

Although decay by contact interaction dominates for $\Lambda=m_{\star}$, the decay via gauge interactions is proportional to $m_{\star}^{3} / \Lambda^{2}$, while decay via contact interactions varies as $m_{\star}^{5} / \Lambda^{4}$. Therefore, the relative importance of the decay mediated by contact interactions on the total decay width will be suppressed by the factor $\left(m_{\star} / \Lambda\right)^{2}$. This fact is clearly illustrated in Table 1 One can see that ( contrary to $\Lambda=m_{\star}$ ) in the case $\Lambda=6 \mathrm{TeV}$ the excited electron decay is dominated by gauge interactions up to $m_{\star} \leq 1.5 \mathrm{TeV}$. However, for $m_{\star} \geq 3 \mathrm{TeV}$ the contribution from contact interactions to the total decay width is dominant and cannot be neglected. Decays by contact interactions yield $e j j$ and ell final states, but the two jets or the two leptons accompanying the electron do not have the mass of the $Z$.

Table 1. Decay widths $(\Gamma)$ and branching ratios via gauge and contact interactions, for $\Lambda=m_{\star}$ and $\Lambda=6 \mathrm{TeV}$ (in parenthesis )

\begin{tabular}{cccc}
\hline$m_{\star}(\mathrm{GeV})$ & $\Gamma_{\text {tot }}(\mathrm{GeV})$ & $\Gamma_{G} / \Gamma_{\text {tot }}$ & $\Gamma_{C} / \Gamma_{\text {tot }}$ \\
\hline 1000 & $89.9(0.26)$ & $0.08(0.75)$ & $0.92(0.25)$ \\
\hline 3000 & $270(20.8)$ & $0.08(0.25)$ & $0.92(0.75)$ \\
\hline 5000 & $451(224)$ & $0.08(0.11)$ & $0.92(0.89)$ \\
\hline
\end{tabular}

\section{$3 \quad$ Simulations and results}

Single production of excited electrons and their decays are simulated. The branching ratios in three channels are given in Table 2 as obtained from PYTHIA. The simulations of excited lepton signal and relevant backgrounds were performed with COMPHEP 16, the COMPHEP-PYTHIA interface 17] and PYTHIA 18. programs chain. 
As shown in the previous section, the decay rate of excited leptons via contact interactions is comparable to the one via gauge interaction and can even be the dominant one, contributing up to $92 \%$ to the total width $\left(m_{\star}=6 \mathrm{TeV}\right.$ case). Therefore it is very important to consider gauge and contact decays of the excited electron simultaneously. In present version of PYTHIA only gauge decay of the excited fermions is implemented. The use of PYTHIA only, therefore, for our task would be misleading.

Table 2. Branching ratios of excited lepton decay via gauge and contact interactions.

\begin{tabular}{cccc}
\hline$f=f^{\prime}=1$ & \multicolumn{3}{c}{ Gauge interactions } \\
\hline Decay mode & $\mathrm{e}^{\star} \rightarrow \nu \mathrm{W}^{-}$ & $\mathrm{e}^{\star} \rightarrow \mathrm{e} \gamma$ & $\mathrm{e}^{\star} \rightarrow e Z$ \\
\hline$B R$ & 0.604 & 0.283 & 0.113 \\
\hline \multicolumn{4}{c}{ Contact interactions } \\
\hline Decay mode & $\mathrm{e}^{\star} \rightarrow \mathrm{e} \nu \nu$ & $\mathrm{e}^{\star} \rightarrow$ eee & $\mathrm{e}^{\star} \rightarrow e j j$ \\
\hline$B R$ & 0.18 & 0.11 & 0.71 \\
\hline
\end{tabular}

One should also take into account the contact decays of the excited electron and, therefore, apply the proper correction for the gauge decay branching fraction (BF) from PYTHIA. Taking this into account, we have used PYTHIA with proper BF correction to perform decays of excited electrons mediated by gauge interactions, while the consideration of decays of excited leptons via contact interactions were made available with the help of COMPHEP.

The ATLFAST 19 code has been used to take into account the experimental conditions prevailing at LHC for the ATLAS detector. The detector concept and its physics potential have been presented in the Technical Proposal 20 and the Technical Design Report 21. The ATLFAST program for fast detector simulations accounts for most of the detector features: jet reconstruction in the calorimeters, momentum/energy smearing for leptons and photons, magnetic field effects and missing transverse energy. It provides a list of reconstructed jets, isolated leptons and photons. In most cases, the detector dependent parameters were tuned to values expected for the performance of the ATLAS detector from full simulation.

The electromagnetic calorimeters were used to reconstruct the energy of leptons in cells of dimensions $\Delta \eta \times \Delta \phi=0.025 \times 0.025$ within the pseudorapidity range $-2.5<\eta<2.5 ; \phi$ is the azimuthal angle. The electromagnetic energy resolution is given by $0.1 / \sqrt{E}(G e V) \bigoplus 0.007$ over this pseudorapidity $(\eta)$ region. The electromagnetic showers are identified as leptons when they lie within a cone of radius $\Delta R=\sqrt{(\Delta \eta)^{2} \times(\Delta \phi)^{2}}=0.2$ and possess a transverse energy $E_{T}>5$ $\mathrm{GeV}$. Lepton isolation criteria were applied, requiring a distance $\Delta R>0.4$ from other clusters and maximum transverse energy deposition, $E_{T}<10 \mathrm{GeV}$, in cells in a cone of radius $\Delta R=0.2$ around the direction of electron emission.

It must be mentioned that standard parametrization in the ATLFAST has been used for the electron resolution but detailed studies are needed, using test beam data and GEANT full simulation to validate the extrapolation of the resolution function to electron energies in the $\mathrm{TeV}$ range. 


\section{$3.1 \quad e^{\star} \rightarrow e \gamma$ Channel}

The production cross sections for the excited electrons and backgrounds are given in Tables 3 and 4 respectively.

In order to enrich the event statistics in the region of high invariant masses, simulated background events were generated separately in different regions of the reaction transverse momentum, $\hat{p}_{T}$.

Several types of backgrounds were selected for comparison with the signal events.

The natural background for the signal process studied is the process $q \bar{q} \rightarrow$ $Z+\gamma$, where $Z$ decays to two leptons (electron - positron pair) yielding two leptons and a photon in the final state.

Another type of background process is the single production of $Z$ plus jet, where $Z$ decays to the lepton pair and the additional jet in the event could be misidentified with a photon. However, the photon/jet separation studies [21] done in ATLAS give a jet total rejection of around 900 for jet $E_{T}$ around of 20 $\mathrm{GeV}$ at high luminosity $\left(10^{34} \mathrm{~cm}^{-2} \mathrm{~s}^{-1}\right)$. It is expected that the rejection factor will be at least as high at higher energies.

Table 3. Cross section $\times \mathrm{BR}(\mathrm{pb})$ (PYTHIA) for $q \bar{q} \rightarrow e^{*} e \rightarrow e^{+} e^{-} \gamma$, scale $\Lambda=m_{\star}$ and couplings $f=f^{\prime}=1$.

\begin{tabular}{ccccccc}
\hline$m_{\star}(\mathrm{GeV})$ & 500 & 1000 & 2000 & 3000 & 4000 & 5000 \\
\hline$q \bar{q} \rightarrow e^{*} e \rightarrow e^{+} e^{-} \gamma$ & 2950. & 87.3 & 1.35 & $6.5 \times 10^{-2}$ & $4.25 \times 10^{-3}$ & $3.3 \times 10^{-4}$ \\
\hline
\end{tabular}

Decays of excited electrons produce characteristic event topologies and signatures which allow one to distinguish them from their backgrounds. Due to their large mass, excited electrons would produce events with large transverse energy. We study the event topology of excited electrons in the decay channel $e^{\star} \rightarrow e \gamma$ and apply the selection criteria for both signal and background events.

The following cuts were used to enhance the signal:

- The transverse momentum of leptons was required to be at least $100 \mathrm{GeV}$.

Table 4. Cross section $\sigma(\mathrm{pb})$ from PYTHIA for the total background over various $\hat{p}_{T}(\mathrm{TeV})$ ranges

\begin{tabular}{ccc}
\hline$\hat{p}_{T}$ & $\gamma+Z$ & $Z+j e t$ \\
\hline $0.1<\hat{p}_{T}<0.3$ & $5.2 \times 10^{-2}$ & 27.71 \\
$0.3<\hat{p}_{T}<0.6$ & $1.3 \times 10^{-3}$ & $5.1 \times 10^{-1}$ \\
$0.6<\hat{p}_{T}<1.0$ & $7.4 \times 10^{-5}$ & $2.0 \times 10^{-2}$ \\
$\hat{p}_{T}>1.0$ & $5.5 \times 10^{-6}$ & $6.4 \times 10^{-3}$ \\
\hline
\end{tabular}




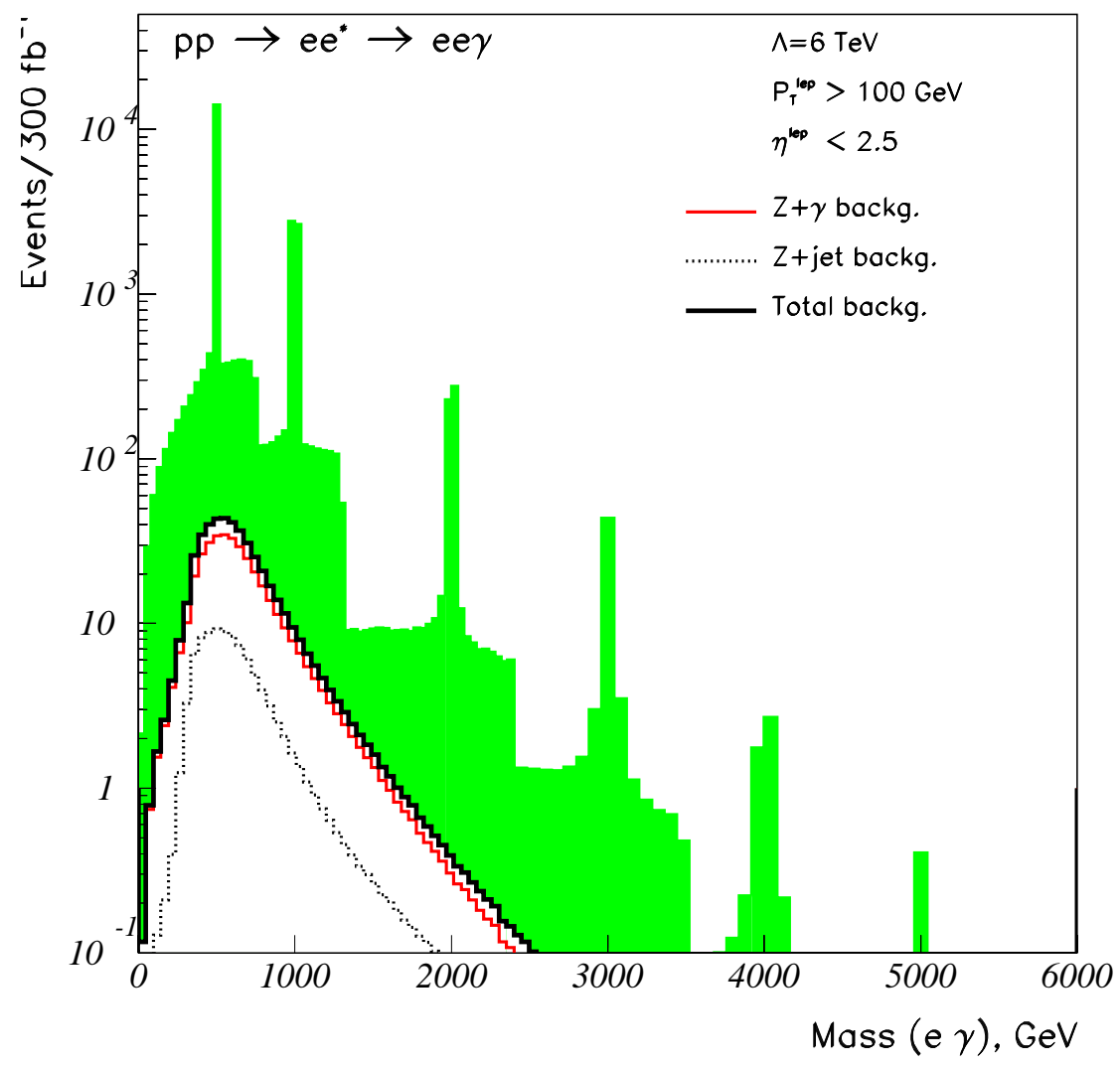

Fig. 4. Invariant mass distributions of $e \gamma$ for various excited lepton masses for an integrated luminosity of $300 \mathrm{fb}^{-1}$

- Electrons were required to be within pseudorapidity acceptance of the ATLAS tracker and precise calorimetry, i.e. $|\eta|<2.5$.

The resulting invariant mass distributions of photon - electron pairs are presented in Fig. प प for different $m_{\star}$ masses of the excited lepton for $\Lambda=6 \mathrm{TeV}$. The resonances are clearly seen above the combinatorial and other backgrounds. The distributions were normalized to an integrated luminosity of $L=300 \mathrm{fb}^{-1}$.

The signal significances are defined at each mass point as $S / \sqrt{B}$ where $S$ and $B$ are the number of accepted signal and background events in the selected mass bin width ( $\Delta M)$, respectively. For the mass bin width the value was taken equal to $\pm 2 \sigma$ width of the invariant mass distribution around the excited electron peak. A modest rejection factor of 1000 21 has been used for jets mistagged as photons to suppress the $Z+$ jet background. Achievable mass limits for the 
Table 5. Signal significance $S / \sqrt{B}, S$ for signal, $B$ for total background, and number of events are calculated in the window $\Delta M$ around the excited electron peak for an integrated luminosity of $L=300 \mathrm{fb}^{-1}$ and $\Lambda=6 \mathrm{TeV}$.

\begin{tabular}{llllll}
\hline$m_{\star}(\mathrm{TeV}) \rightarrow$ & 0.5 & 1.0 & 2.0 & 3.0 & 4.0 \\
\hline$\Delta M, \mathrm{GeV}$ & 16 & 30 & 50 & 80 & 120 \\
\hline$S$ & 13700 & 5281 & 490 & 43 & 5.0 \\
\hline$S / \sqrt{B}$ & 2385 & 2104 & 1044 & 311 & 66 \\
\hline
\end{tabular}

excited single electron production are established by requiring that at least 5 events survive the cuts and $S / \sqrt{B}>5$.

As can be seen from Table 5 the masses achieved for excited single lepton in the photon and electron decay modes are $\sim 4.0 \mathrm{TeV}$ for $\Lambda=6 \mathrm{TeV}$, the couplings $f=f^{\prime}=1$ and for an integrated luminosity $L=300 \mathrm{fb}^{-1}$ at CERN LHC.

\section{$3.2 e^{\star} \rightarrow Z e /$ eejj(eeee) channels: combining gauge and contact decays}

Here we consider the decay of excited electrons to $Z e$ mediated by gauge interactions together with the 3-body decay $(e l l, e j j)$ of the excited electron mediated by contact interactions. It is natural to treat those decays together since we consider the vector boson decay modes to $Z \rightarrow e e$ or $Z \rightarrow q q$, and the signal consists of four electrons or two jets and two electrons. The decay channel $Z \rightarrow$ $\mu \mu$ is expected to give results comparable to $Z \rightarrow e e$, but with somewhat worse energy resolution.

We will allow for the possibility that one of the electrons could be outside the acceptance region of the detector or could be lost by some inefficiency of reconstruction.

The dominant backgrounds for the signal process studied when $Z$ decays to an electron-positron pair are:

- $q \bar{q} \rightarrow Z+\gamma$, where $Z$ decays to two leptons (electron - positron pair) yielding two leptons and a photon in the final state. Good rejection of photons in electron reconstruction could help to suppress this source of background.

- $Z+Z$ production, where both $Z$ decay to an electron and a positron.

- $W+Z$ production, where $Z$ again decays to an electron and a positron, and $W$ decays to an electron and a neutrino.

In the case when $Z$ decays to jets the following backgrounds could be relevant:

- $Z+$ jet production, where a $Z$ decay to an electron and a positron, and additional jet produced by means of the final state radiation mechanism. 
- $Z+Z$ production, where one of $Z$ decays to an electron-positron pair, while the other $Z$ decays to jets.

- $q \bar{q} \rightarrow Z+\gamma$, where $Z$ decays to two jets yielding two jets and a photon in the final state. Again, as in the case of $Z \rightarrow e e$ decay, good rejection of photons in electron reconstruction could help to suppress this source of background.

The production cross sections for the signal channels and backgrounds are given in Table [6 and Table [7 respectively.

Table 6. Cross section $\times \mathrm{BR}(\mathrm{pb})$ from PYTHIA for $e^{*} e \rightarrow Z e e$, scale $\Lambda=m_{\star}$ and couplings $f=f^{\prime}=1$.

\begin{tabular}{cccccc}
\hline$m_{\star}(\mathrm{GeV})$ & 500 & 1000 & 2000 & 3000 & 4000 \\
\hline $\mathrm{Z} \rightarrow \mathrm{ee}$ & 38.5 & 1.2 & $1.9 \times 10^{-2}$ & $8.5 \times 10^{-4}$ & $5.5 \times 10^{-5}$ \\
$\mathrm{Z} \rightarrow \mathrm{jj}$ & 800. & 24.4 & $3.8 \times 10^{-1}$ & $1.8 \times 10^{-2}$ & $1.2 \times 10^{-3}$ \\
\hline
\end{tabular}

Table 7. Cross section $\sigma(\mathrm{pb})$ from PYTHIA for the total background with $Z$ $\rightarrow e e(j j)$ over given $\hat{p}_{T}(\mathrm{TeV})$ ranges

\begin{tabular}{cccc}
\hline$Z \rightarrow e e$ & & & \\
\hline$\hat{p}_{T}$ & $Z+\gamma$ & $Z+Z$ & $Z+W$ \\
\hline $0.1<\hat{p}_{T}<0.3$ & $5.2 \times 10^{-2}$ & $1.7 \times 10^{-3}$ & $1.1 \times 10^{-2}$ \\
$\hat{p}_{T}>0.3$ & $1.4 \times 10^{-3}$ & $5.8 \times 10^{-5}$ & $3.0 \times 10^{-4}$ \\
\hline \hline$Z \rightarrow j j$ & & & \\
\hline$\hat{p}_{T}$ & $Z+j e t$ & $Z+\gamma$ & $Z+Z$ \\
\hline $0.1<\hat{p}_{T}<0.3$ & 21.55 & $9.7 \times 10^{-1}$ & $7.4 \times 10^{-1}$ \\
$\hat{p}_{T}>0.3$ & $4.0 \times 10^{-1}$ & $2.7 \times 10^{-2}$ & $2.6 \times 10^{-2}$ \\
\hline
\end{tabular}

The following cuts were used to separate the signal from background:

- eeee final state case

- The transverse momenta of leptons were required to be at least 60 $\mathrm{GeV}$.

- Electrons were required to be within pseudorapidity acceptance of the ATLAS tracker and precise calorimetry, i.e. $|\eta|<2.5$.

- eejj final state case

- The transverse momenta of jets and leptons were required to be at least $120 \mathrm{GeV}$.

- Electrons were required to be within pseudorapidity acceptance of the ATLAS tracker and precise calorimetry, i.e. $|\eta|<2.5$. 
- The cosine of the opening angle between an electron and a jet was required to be greater than -0.8 .

The resulting invariant mass distributions of three lepton system are presented in Fig. 5 for different $m_{\star}$ masses of the excited lepton. The four electrons with highest $p_{T}$ were selected for the reconstruction. As before, a scale $\Lambda=6$ $\mathrm{TeV}$ is taken as reference. A photon/electron rejection factor of 500 21] has been used to suppress the $Z+\gamma$ background. The distributions were normalized to an integrated luminosity of $L=300 \mathrm{fb}^{-1}$.

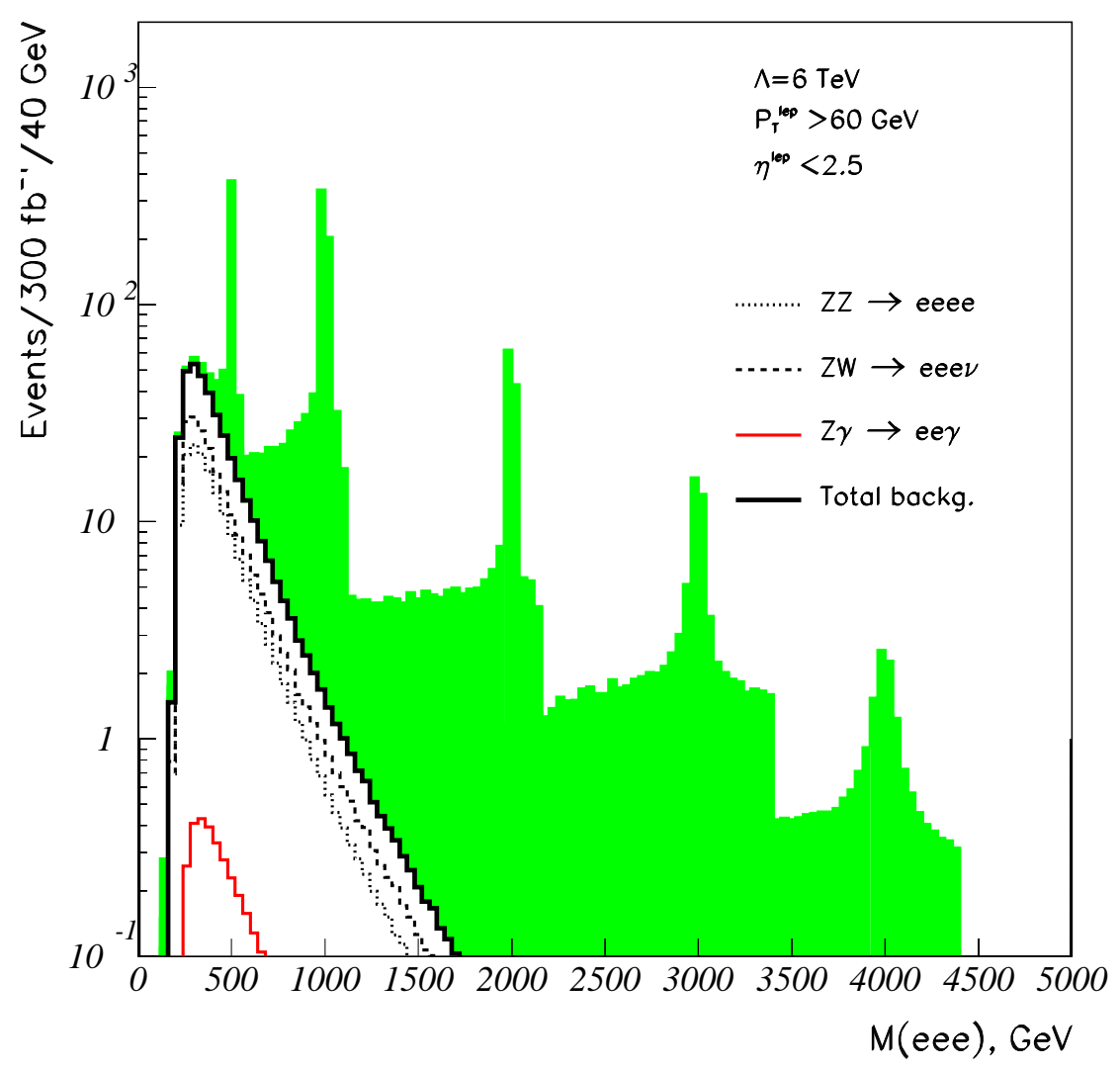

Fig. 5. Invariant mass distributions of combined $Z e(Z \rightarrow$ ee $)+e e e e$ contributions from gauge and contact excited lepton decays for masses of $500 \mathrm{GeV}$, $1 \mathrm{TeV}, 2 \mathrm{TeV}, 3 \mathrm{TeV}$ and $4 \mathrm{TeV}$ for an integrated luminosity of $300 \mathrm{fb}^{-1}$ and $\Lambda=6 \mathrm{TeV}$.

For a $Z$ boson that decays hadronically, the resulting jets are highly boosted for large values of $m_{\star}$. If the two jets were merged as a single jet, the mass of 
the excited lepton was reconstructed as the invariant mass of the jet-electron system.

The resulting invariant mass distributions of a lepton and a jet pair are presented in Fig. [6 for different $m_{\star}$ and $\Lambda=6 \mathrm{TeV}$. The same photon/electron rejection factor of 500 has been used to suppress the $Z+\gamma$ background. The distributions were normalized to an integrated luminosity of $L=300 \mathrm{fb}^{-1}$. For $m_{\star}=500 \mathrm{GeV}$ we have the peak falling on top of background. However, this is not the problem, since signal to background ratio is about one. So, for the signal which is about the same as the background the problem of understanding of K-factor does not occur. We believe that by the time of LHC, uncertainties for the $W j j$ cross section will be known far better then $50 \%$.

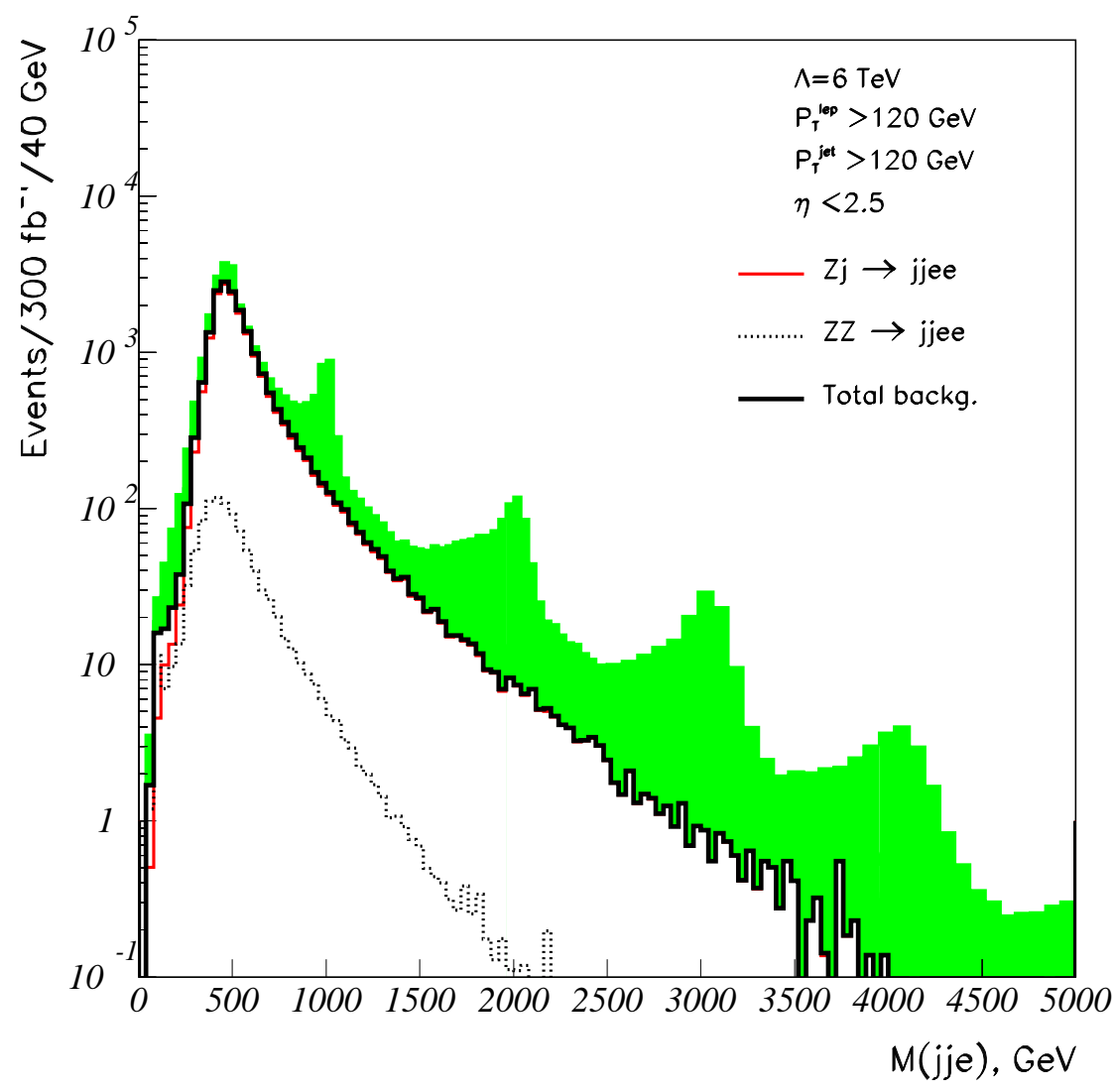

Fig. 6. Invariant mass distributions of $Z e(Z \rightarrow \mathrm{jj})+e e j j$ of combined contributions from gauge and contact excited lepton decays for masses of 500 $\mathrm{GeV}, 1 \mathrm{TeV}, 2 \mathrm{TeV}, 3 \mathrm{TeV}$ and $4 \mathrm{TeV}$ for an integrated luminosity of $300 \mathrm{fb}^{-1}$ and $\Lambda=6 \mathrm{TeV}$. 
The signal significances are defined at each mass point as $S / \sqrt{B}$ where $S$ and $B$ are the number of accepted signal and background events in the selected mass bin, respectively. Achievable mass limits for the excited single electron production are established by requiring that at least 5 events survive the cuts and $S / \sqrt{B}>5$.

As can be seen from Table 8 and Fig. 7 the masses achieved for excited single lepton in the $Z e$ decay modes are $\sim 3.0 \mathrm{TeV}$ for $\Lambda=6 \mathrm{TeV}$, the couplings $f=f^{\prime}=1$ and for an integrated luminosity $L=300 \mathrm{fb}^{-1}$ at CERN LHC. Due to a low number of events in the signal events sample, we do not present the data for the masses beyond $4 \mathrm{TeV}$, but with an extended data taking period, this region of masses may also be reachable.

Table 8. Signal significance $S / \sqrt{B}, S$ for signal, $B$ for total background, and number of events are calculated for an integrated luminosity of $L=300 \mathrm{fb}^{-1}$ and $\Lambda=6 \mathrm{TeV}$ within selected mass bin width $(\Delta M)$.

\begin{tabular}{llllll}
\hline$m_{\star}(\mathrm{TeV}) \rightarrow$ & 0.5 & 1.0 & 2.0 & 3.0 & 4.0 \\
\hline$q \bar{q} \rightarrow e^{\star} e \rightarrow$ eeee & & & & & \\
\hline$\Delta M, \mathrm{GeV}$ & 20 & 38 & 63 & 84 & 120 \\
\hline$S$ & 168 & 192 & 46 & 14 & 3.4 \\
\hline$S / \sqrt{B}$ & 53 & 135 & 145 & 99 & 76 \\
\hline \hline$q \bar{q} \rightarrow e^{\star} e \rightarrow e e j j$ & & & & & \\
\hline$\Delta M, \mathrm{GeV}$ & 40 & 60 & 106 & 180 & 200 \\
\hline$S$ & 2102 & 2415 & 636 & 176 & 24 \\
\hline$S / \sqrt{B}$ & 30 & 120 & 105 & 69 & 30 \\
\hline
\end{tabular}

\section{$3.3 \quad e^{\star} \rightarrow W \nu$ Channel}

The production cross sections for this channel are given in Table 9

Table 9. Cross section $\times \mathrm{BR}(\mathrm{pb})$ (PYTHIA) for $e^{*} e \rightarrow W+\nu e$, scale $\Lambda=m_{\star}$ and couplings $f=f^{\prime}=1$.

\begin{tabular}{cccccc}
\hline$m_{\star}(\mathrm{GeV})$ & 1000. & 2000. & 3000. & 4000. & 5000. \\
\hline$W \rightarrow j j$ & 93.5 & 1.1 & $3.9 \times 10^{-2}$ & $2.0 \times 10^{-3}$ & $1.3 \times 10^{-4}$ \\
$W \rightarrow e \nu$ & 14.8 & 0.2 & $6.0 \times 10^{-3}$ & $3.3 \times 10^{-4}$ & $2.0 \times 10^{-5}$ \\
\hline
\end{tabular}

In the subprocesses involving a $W$ decaying hadronically, the final state consists of two jets, an electron and a neutrino. An attempt to reconstruct the mass of $(j j \nu)$ system is presented in Fig. 8 for excited electron masses of 500, 750, 1000, 1250 and $1500 \mathrm{GeV}$ for an integrated luminosity of $300 \mathrm{fb}^{-1}$ and $\Lambda=6 \mathrm{TeV}$. 

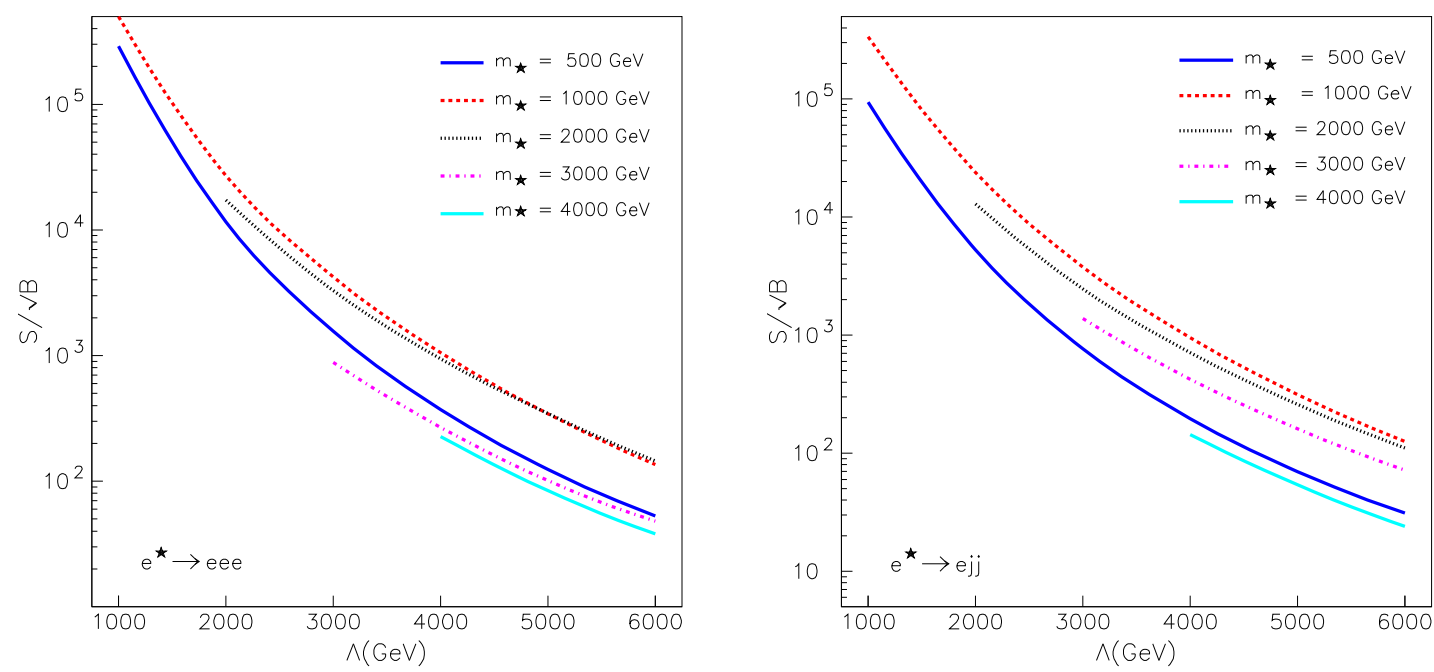

Fig. 7. Excited electron signal significance shown for different scale $\Lambda$ at an integrated luminosity at LHC $L=300 \mathrm{fb}^{-1}$.

The two jets with highest $p_{T}$ were used for the mass reconstruction. Because the reconstructed mass cannot account for the longitudinal missing momentum, a wide distribution is obtained.

The plots were obtained using following cuts:

- The transverse momenta of jets were required to be at least $70 \mathrm{GeV}$.

- The transverse momentum of electron was required to be $200 \mathrm{GeV}$ within pseudorapidity $|\eta|<2.5$ for all excited lepton masses considered.

- It was required to have a reconstructed $W \rightarrow j j$ with a mass in the $70-90$ $\mathrm{GeV}$ range in the event to be selected.

- The missing $p_{T}$ cut was required to be $200 \mathrm{GeV}$.

As can be seen from Fig. 8 signal signatures can be observed for excited electron masses from $500 \mathrm{GeV}$ up to $1000 \mathrm{GeV}$ over the total background.

The second subprocess, when $W$ decays to a lepton and a neutrino, gives pairs of leptons and neutrinos. The effective mass reconstruction is very difficult in this case. It requires the evaluation of the neutrino longitudinal momenta, which is impossible without certain kinematical constraints. However, estimating the acceptance and efficiencies at $65 \%$ and $20 \%$, respectively, the transverse mass distribution $m_{T}=\sqrt{\left|\mathbf{p}_{T}(e)\right|^{2}+\left|\mathbf{p}_{T}(\ell)+\mathbf{p}_{T}^{\text {miss }}\right|^{2}}(e$ is the electron recoiling against $e^{*}$ and $\ell$ is the lepton from $W$ decay) should yield an observable excess of events over backgrounds for excited electron masses up to $4.5 \mathrm{TeV}$. 


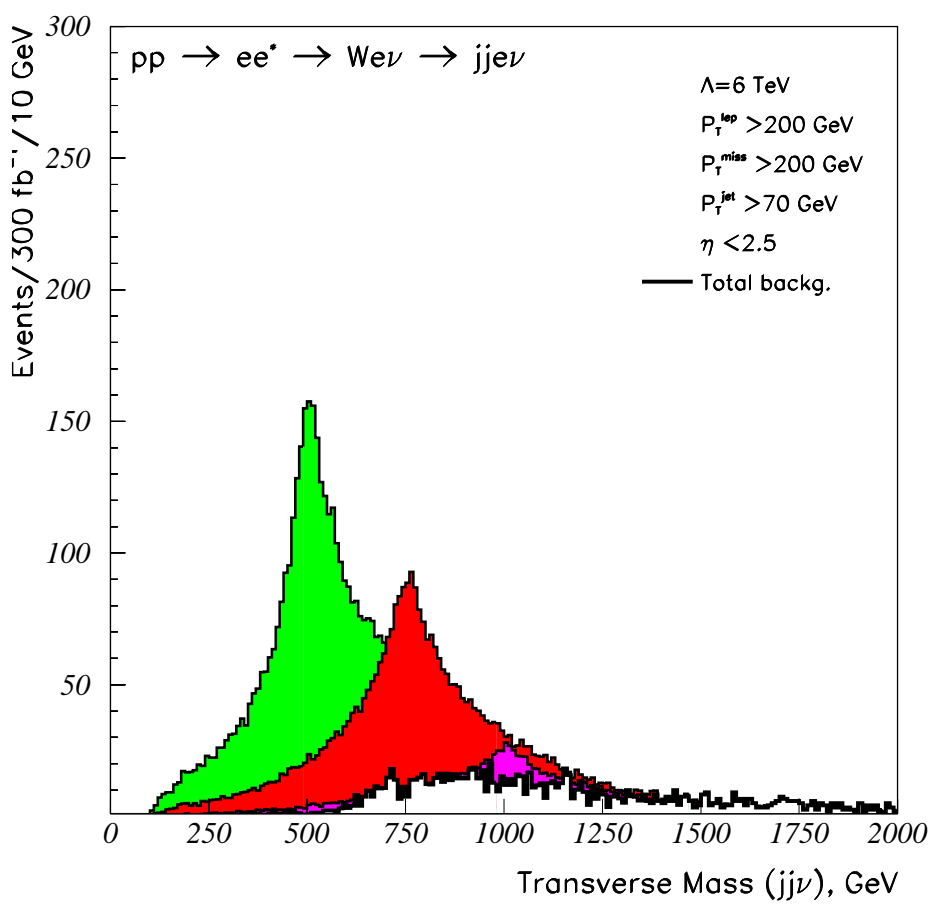

Fig. 8. Invariant mass distribution of $(j j \nu)$ system for excited lepton masses of $500,750,1000$ and $1250 \mathrm{GeV}$ for an integrated luminosity of $300 \mathrm{fb}^{-1}$ and $\Lambda=6$ $\mathrm{TeV}$. The total background from $W+$ jet and $W+W$ production is also shown.

This process could therefore confirm excited electron observation, obtained from others channels.

\section{Conclusions}

In the framework of a composite model of quarks and leptons, sharing common constituents, excited electrons could be produced copiously at CERN LHC. The first indication for the excited electrons could be the production of ordinary leptons at rates much larger than expected in the framework of the SM. Large lepton yields are expected if quarks and leptons share common sub-constituents. Clean signatures are predicted by the large fraction of decays with leptons in the final state, that are a consequence of the uniform coupling among quarks and leptons through contact interactions. We have presented the results of excited single electron production with subsequent decay mediated by gauge and contact interactions. Singly produced excited electrons could be accessible up to a mass of $5 \mathrm{TeV}$ at LHC for $\Lambda=6 \mathrm{TeV}$. For the excited electrons masses decay both 
contact and gauge interactions plays important role and should be considered together. For comparatively light excited leptons $(<1 \mathrm{TeV})$ the decays mediated by contact interactions may be considerably suppressed, while for higher masses of excited states the contribution from contact interactions to the total decay width is dominant. The decay channel $Z \rightarrow \mu \mu$ is expected to give results comparable to $Z \rightarrow e e$, but with somewhat lower energy resolution.

\section{Acknowledgments}

This work has been performed within the ATLAS Collaboration with the help of the simulation framework and tools which are the result of the collaborationwide efforts. We would like also to thank G. Azuelos, H.Baer, D. Froidevaux, F. Gianotti, I. Hinchliffe, L. Poggioli, L.Reina for their comments about the subject. C.L. and R.M. thank NSERC/Canada for their support.

\section{References}

1. H.Terazawa, M.Yasue, K.Akama, M.Hayashi: Phys.Lett. B 112 (1982) 387 F.M.Renard: Il Nuovo Cimento, 77A (1983) 1

A. De Rujula, L.Maiani, and R.Petronzio: Phys.Lett. B 140 (1984) 253

E.J.Eichten, K.D.Lane, and M.E.Peskin: Phys.Rev. D 50 (1983) 811

2. H.Terazawa, Y.Chikashige, and K.Akama: Phys.Rev. D 15 (1977) 480

3. Y.Ne'eman: Phys.Lett. B 82 (1979) 69

4. U.Baur, M.Spira, and P.M.Zerwas: Phys. Rev. D 42 (1990) 815

5. R.Barate et al.: Eur.Phys.Jour. C 12 (2000) 183

6. B.Abbott et al.: Phys.Rev.Lett. 82 (1999) 4769

7. J.Breitweg et al.: Z.Phys. C 76 (1997) 631

8. G.Abbiendi et al.: Phys.Lett. B 465 (1999) 303

9. O.Çakır, R.Mehdiyev: Phys.Rev. D 60 (1999) 034004

10. O.Çakır, C.Leroy, and R.Mehdiyev: Phys.Rev. D 62 (2000) 114018

11. O.Çakır, C.Leroy, and R.Mehdiyev: Phys.Rev. D 63 (2001) 094014

12. H.L.Lai et al., CTEQ Coll.: Eur.Phys.J C 12 (2000) 375

13. M.Glúck, E.Reya and A.Vogt: Z.Phys C 53 (1992) 651

14. H.L.Lai et al., CTEQ Coll.: Phys.Rev. D 51 (1995) 4763

15. O.J.Eboli, S.M.Lietti and P.Mathews: Phys.Rev. D 65 (2002) 075003

16. A.Pukhov at al., hep-ph/9908288 (1999)

17. A.S.Belyaev et al., hep-ph/0101232

18. T.Sjostrand: Computer Phys. Communications 82 (1994) 74

19. E.Richter-Was, D.Froidevaux and L.Poggioli: ATLAS Note PHYS-98-131 (1998)

20. Technical Proposal, ATLAS Coll.: Report No. CERN/LHCC/94-43

21. ATLAS Detector and Physics Performance Technical Design Report. Vol. 1 CERN/LHCC/99-14, (1999)

22. ATLAS Detector and Physics Performance Technical Design Report. Vol. 2 CERN/LHCC/99-15, (1999); E.C.Katsoufis: ATLAS Note PHYS-NO-038 (1994) 